\title{
Effect of Vitamin D Supplementation on Intracytoplasmic Sperm Injection Outcomes: A Randomized Double-Blinded Placebo-Controlled Trial
}

\author{
Ahmed H. Abd-Ellah'1, Abd El-Naser Abd El-Gaber Ali ${ }^{1 *}$, Mustafa M. Khodry', \\ Mahmoud I. El-Rasheedy ${ }^{2}$, Ahmed M. Abbas ${ }^{3}$ \\ ${ }^{1}$ ART Unit, Department of Obstetrics \& Gynecology, Faculty of Medicine, South Valley University, Qena, Egypt \\ ${ }^{2}$ Department of Obstetrics \& Gynecology, Faculty of Medicine, Al-Azhar University, Assiut, Egypt \\ ${ }^{3}$ Department of Obstetrics \& Gynecology, Faculty of Medicine, Assiut University, Assiut, Egypt \\ Email: *abdelnaser.abdelgaber@med.svu.edu.eg
}

How to cite this paper: Abd-Ellah, A.H., Ali, A.E.-N.A.E.-N., Khodry, M.M., El-Rasheedy, M.I. and Abbas, A.M. (2018) Effect of Vitamin D Supplementation on Intracytoplasmic Sperm Injection Outcomes: A Randomized Double-Blinded Placebo-Controlled Trial. Open Journal of Obstetrics and Gynecology, 8, 1549-1556.

https://doi.org/10.4236/ojog.2018.814155

Received: November 29, 2018

Accepted: December 18, 2018

Published: December 21, 2018

Copyright $(9) 2018$ by authors and Scientific Research Publishing Inc. This work is licensed under the Creative Commons Attribution International License (CC BY 4.0).

http://creativecommons.org/licenses/by/4.0/

\begin{abstract}
OBJECTIVE: To assess the effect of vitamin D supplementation on enhancement of embryos implantation rates in intracytoplasmic sperm injection procedures. STUDY DESIGN: A randomized double-blinded controlled trial. SETTING: Assisted Reproduction Unit of Obstetrics and Gynecology Department, Faculty of Medicine, South Valley University, Egypt. DURATION: From December $1^{\text {st }} 2017$ to the end of October 2018. SUBJECTS and METHDS: Eighty infertile couples that underwent ICSI procedure were entrapped in this study. Patients were divided into two groups; Group I (Vitamin D group) $=40$ patients (received vitamin D supplementation in the form of Vidrop oral drops $600 \mathrm{IU} /$ day starting after ovum pickup) and Group II (placebo group) $=40$ patients (received a placebo "normal saline oral drop"). RESULTS: There was a significantly increased embryo implantation rates $(53.2 \%$ in vitamin D group versus $46.7 \%$ in placebo group), there was a mild statistically significant difference in the clinical pregnancy rates $(45.9 \%$ in vitamin D group versus $39.4 \%$ in placebo group) with p-value $<0.05$ and there was an improvement in ongoing pregnancy rate as there was a mild statistically significant difference in first trimester miscarriage rates $(12.5 \%$ in vitamin D group versus $17.5 \%$ in placebo group) with $\mathrm{p}$ value $<0.05$ CONCLUSIONS: Vitamin D supplementation could improve embryos implantation rates and rate of ongoing pregnancy by decline in the first trimester miscarriage rate.
\end{abstract}

\section{Keywords}

Vitamin D, Intracytoplasmic Sperm Injection, ICSI 


\section{Introduction}

Infertility is considered the most common disease of reproductive-age, affecting $15 \%$ to $25 \%$ of all couples who try to have a baby in western countries [1]. Assisted reproductive technology (ART) has emerged as the most effective treatment modality to overcome infertility problem and currently is responsible for the birth of more than 50,000 babies in the United States alone [2] and about 140,000 babies in Europe yearly [3]. So all the time there are many trials of studies to identify the potentially modifiable predictors of the successfully treatment method, including patient diet regimen and her lifestyle.

Vitamin D has a common name "sunshine hormone", that is a fat soluble hormone which has an integral part in calcium \& phosphorous homeostasis and has a role in maintaining healthy bone and teeth, also it is involved in providing the protection against a large number of diseases like cancer, multiple sclerosis, diabetes, obesity, cardiovascular diseases and many other diseases; also it is thought to have a role in infertility treatment [4].

Vitamin D deficiency had been advocated as a possible reason of infertility in many previous studies in literature for the past several years [5]. In rodent models, vitamin D deficiency declines fertility rates in females as a result of hypoplastic uterus, impaired follicular growth, and chronic anovulation [6]. Vitamin D biological actions are mediated through the receptors of vitamin $\mathrm{D}$ which has been detected in intestines, parathyroid glands, the skeleton and reproductive organs involving the ovary, uterus, placenta, and pituitary gland [7].

In many studies, vitamin D deficiency was highly prevalent in infertile women with polycystic ovary syndrome (PCOS) [8]. It has been postulated that vitamin D may play an important role in the process of embryos implantation where vitamin $\mathrm{D}$ enzymes and vitamin $\mathrm{D}$ receptors have been detected in the endometrial tissues [9]. Vitamin D deficiency had been linked to poor and abnormal placentation that lead to hypertensive disorders of pregnancy and intrauterine growth restriction of the fetus [10]. Vitamin D can modulate the inflammatory and proliferation process in endometriotic cells, and decrease in Vitamin D levels could be associated with development endometriosis, so vitamin D supplementation could be a novel therapeutic strategy for managing endometriosis [11].

\section{Aim of Work}

Primary outcome, was to assess the effect of vitamin D supplementation in enhancement of implantation rates in intracytoplasmic sperm injection (ICSI) cycles.

Secondary outcome, was to assess the effect of vitamin D in minimize first trimester miscarriage.

\section{Patients and Methods}

Eighty infertile couples underwent ICSI procedures were entrapped in this study 
at assisted reproduction unit of Qena university hospital, South Valley University from December 2017 to October 2018. A verbal counseling followed by a written consent was obtained from each couples participated in this study according to the Medical Ethics committee of Faculty of Medicine, South Valley University. The study was approved by the institutional ethical review board.

Inclusion criteria; Any infertile couples attempted to Assisted Reproduction Technique unit for ICSI procedure.

Exclusion criteria; 1) history of Chronic medical disorder as Chronic renal failure, Renal stones, 2) known cases of chronic vitamin D deficiency, 3) congenital uterine malformations or any uterine cavity lesion, 4) hypersensitivity to vitamin D supplements. For each patient subjected to this study had the following evaluations a) Full history was taken and complete clinical examination was done. b) Ovarian reserve testing (serum AMH, basal serum FSH, basal AFC by transvaginal ultrasound). c) Uterine cavity evaluation (by 3-dimentional transvaginal ultrasound and or office hysteroscopy). d) Routine laboratory investigations (CBC, blood grouping and Rh typing, liver function tests, kidney function tests, prothrombin time, prothrombin concentration, blood sugar). e) Semen analysis.

\subsection{Methodology}

Patients were divided into two groups; Group I (Vitamin D group) $=40$ patients (received vitamin D supplementation in the form of Vidrop oral drops 600 IU/day "6 oral drops". The vitamin D supplementation had started after the day of oocytes retrieval) and Group II (placebo group) $=40$ patients (received a placebo "normal saline oral drop") also placebo administration had started after day of oocytes retrieval. Closed bags prepared and labeled as bags A (Vitamin D group) contained Vitamin D, and bags B (placebo group). Providers and patients had been blinded to the contents of the bags till the study was ended. ICSI procedure was done for all cases and only 1 - 2 embryos (day 3 and day 5) had been allowed to be transferred to each women according to the degree of embryos qualities, the remained good quality embryos were frozen for subsequent transfer in non-stimulated cycles in future with couples permissions.

Hormonal support for luteal phase started in day of ova pick up till pregnancy test time ( 2 weeks post embryos transfer), for positive pregnancy test cases, luteal support continued for the next 3 months. Positive pregnancy test cases had to continue vitamin D supplementation up to the end first trimester of pregnancy. Pregnancy was evaluated by transvaginal ultrasound (SAMSUNG, Korea), in first trimester for confirmation of presence and number of gestational sacs, viability and exclude missed and ectopic pregnancies.

\subsection{Assignment and Masking}

Women were randomized in a 1:1 ratio according to a computer - generated randomization list generated by a research nurse not involved in their clinical management. The hospital pharmacy packaged Vitamin D and identical placebo 
according to the randomization list and labeled the drug packs with subject numbers only. Physicians, research nurses and study subjects were all blinded to the assignment.

\subsection{Statistical Analysis}

Analysis and statistical presentation of this study were conducted, using the mean, standard error, unpaired student t-test, and chi-square tests using the Statistical Program for Social Sciences (SPSS Inc., Version 21.0, Chicago, IL, USA). A two sided $P<0.05$ was considered to be statistically significant.

\section{Results}

There was no statistically significant difference between group I and group II in patient baseline characteristics, duration and doses of controlled ovarian stimulation, retrieved oocytes number, fertilization rate, qualities of embryo or endometrial thickness with p value $>0.05$ (Table $1 \&$ Table 2 ).

There was a significantly increased the embryo implantation rates $(53.2 \%$ in vitamin D group versus $46.7 \%$ in placebo group), there was a mild statistically significant difference in the clinical pregnancy rates between the two groups (45.9\% in vitamin D group versus $39.4 \%$ in placebo group) with p-value $<0.05$ and there was an improvement in ongoing pregnancy rate as there was a mild statistically significant difference in first trimester miscarriage rates $(12.5 \%$ in vitamin $\mathrm{D}$ group versus $17.5 \%$ in placebo group) with $\mathrm{p}$ value $<0.05$ (Figure 1 ).

Table 1. Patient baseline characteristics of the study groups.

\begin{tabular}{cccc}
\hline & $\begin{array}{c}\text { Vitamin D. group } \\
(\mathbf{n}=\mathbf{4 0})\end{array}$ & $\begin{array}{c}\text { Placebo group } \\
(\mathbf{n}=40)\end{array}$ & P-value \\
\hline Age (years) mean \pm SD & $30.80 \pm 4.450$ & $30.25 \pm 4.271$ & $>0.05$ \\
BMI (kg/m 2) mean \pm SD & $25.95 \pm 3.530$ & $25.83 \pm 3.411$ & $>0.05$ \\
AMH (ng/dl) mean \pm SD & $3.20 \pm 2.119$ & $2.36 \pm 1.565$ & $>0.05$ \\
Duration of infertility (years) mean \pm SD & $5.68 \pm 3.851$ & $5.83 \pm 3.876$ & $>0.05$ \\
\hline
\end{tabular}

BMI; body mass index, AMH; anti-mullerian hormone, SD: standard deviation.

Table 2. Comparison between study groups as regarding ICSI cycle characteristics.

\begin{tabular}{cccr}
\hline & $\begin{array}{c}\text { GI (Vitamin D group) } \\
(\mathbf{n}=40)\end{array}$ & $\begin{array}{c}\text { GII (Placebo group) } \\
(\mathbf{n}=40)\end{array}$ & P value \\
\hline Duration of stimulation (days), mean \pm SD & $13.4 \pm 1.3$ & $13.6 \pm 1.6$ & $>0.05$ \\
Dose of gonadotrophins (IU), mean \pm SD & $3982.1 \pm 1371.9$ & $4070.3 \pm 1393.7$ & $>0.05$ \\
N. of oocytes retrieved, mean \pm SD & $12.3 \pm 1.4$ & $13.6 \pm 1.7$ & $>0.05$ \\
Endometrial thickness, mean \pm SD & $10.2 \pm 2.4$ & $10.7 \pm 2.9$ & $>0.05$ \\
Fertilization rate, n (\%) & $87.6 \%$ & $85.9 \%$ & $>0.05$ \\
Embryo quality, n (\%) & & $77.1 \%$ & $>0.05$ \\
A \& B & & $22.9 \%$ & \\
\hline
\end{tabular}




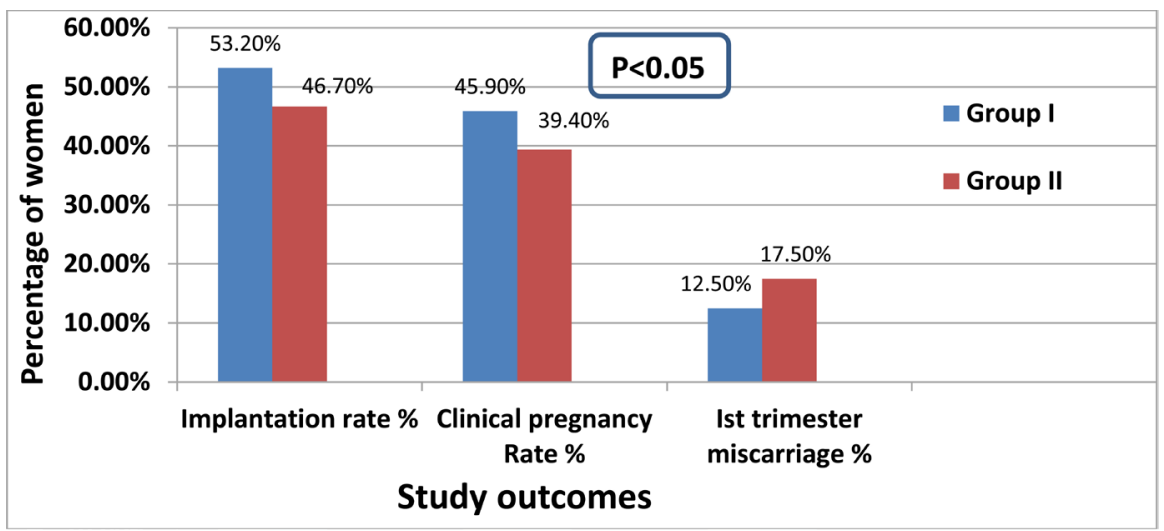

Group I: Vit D. group, Group II: Placebo group.

Figure 1. The clinical outcomes of ICSI cycles among the study groups.

\section{Discussion}

Several lines of evidence suggested that deficiency of vitamin D had a role in disturbances of physiology of the female reproductive system including steroidogenesis in normal fertile women, pathogenesis of the polycystic ovary syndrome (PCOS) cases and endometriosis and in the outcomes of in vitro fertilization (IVF) [12] [13] [14]. The first study researched about vitamin D and its relation to female infertility and its role in-vitro fertilization (IVF) procedures, was done in 1992, and had included only ten cases and it had demonstrated the presence of vitamin D metabolites in the follicular fluid for first time [15]. After that, several studies had been published in this topic with some of them had contradictory results.

Estes et al. had found a decline in the expression of the free form of the vitamin D carrier protein (VDBP) in follicular fluid of cases whose IVF resulted in pregnancy [16]. In the last short period, many studies showed the relation between vitamin D deficiency and infertility [17]. It had been reported that receptors of vitamin D (VDR) are found in human tissues like the reproductive organs of human being and have a major role in enhancement of the biological activity of Vitamin D [18].

In the current study showed no statistically significant differences between vitamin $\mathrm{D}$ group and placebo group as regard to women age, body mass index, $\mathrm{AMH}$, causes and duration of infertility with $\mathrm{p}$ value $>0.05$ (Table 1). Also in this study there were no significant differences between both groups in the gonadotrophins drugs doses, duration of gonadotrophins stimulation, number of retrieved oocytes, and endometrial thickness at time of ovum pickup, fertilization rate or embryo quality (Table 2).

The results of current study showed a positive correlation between vitamin $\mathrm{D}$ supplementation and an increase in implantation rates $53.2 \%$ in Vitamin D group versus $46.7 \%$ in Placebo group). Although our study results was limited due to the small sample size, we had found significantly higher clinical pregnancy rates (45.9\% in Vitamin D group versus 39.4\% in Placebo group) and a lower 
first trimester miscarriage rates in patients received vitamin $\mathrm{D}$ supplementation (12.5\% in Vitamin D group versus $17.5 \%$ in Placebo group) (Figure 1). Many studies in literature had been assessment vitamin D deficiency and its negative impact effect on infertility as general and assisted reproductions results particularly IVF and ICSI procedures from these studies what was reported by Li et al. [19] in California (in 2012) in 1192 of reproductive age women, it had been observed that most of the infertile women had low serum vitamin D levels with $68.6 \%$ cases had levels $<32 \mathrm{ng} / \mathrm{ml}$ and $22.2 \%$ had levels $<20 \mathrm{ng} / \mathrm{ml}$, the study observed that increased BMI of patients, Asian and blacks races were at the highest risk of vitamin D deficiency, especially Asian women.

Gebriel et al. [20] had suggested that females with high levels of 25-hydroxy-vitamin D were significantly higher than those with low levels of vitamin D to achieve clinical pregnancy with ICSI procedure. Rainer et al. [21] (in Oklahoma) retrospectively had evaluated 53 patients had PCOS and had found the deficiency of vitamin D was highly prevalent in these cases, but they had found no positive correlation with Vitamin D levels and the time.

Ozkan et al. [22] study had measured the levels of vitamin D both in the patients serum and in follicular fluid during oocytes retrieval and found the following conclusion, women who had higher levels of vitamin D in their serum and also in the follicular fluid had a significantly achieved pregnancy from IVF comparable with those women who had lower vitamin D levels. These previous studies had been measured vitamin $\mathrm{D}$ levels in both serum and or follicular fluid and supplementation of vitamin $\mathrm{D}$ was only given to patients with low levels of vitamin $\mathrm{D}$, but in our study we had a different policy, we gave patients supplementation of vitamin D prior to embryo transfer in ICSI procedure empirically without measuring their serum or follicular fluid vitamin $\mathrm{D}$ levels in trial to minimize the cost of vitamin D laboratory investigation which is actually has a high cost all over the world. Anyway our results can be discussed because we had positive impacts on embryo implantation and pregnancy outcomes without measuring vitamin D level which could give us a sign that even in patient with normal levels of vitamin D may have benefit from extra supplementation of vitamin D.

\section{Conclusions}

Vitamin D supplementation pre-embryo transfer had;

1) A significant enhanced embryos implantation rate;

2) A significant improvement in ongoing pregnancy by decline rate of first trimester miscarriage.

\section{Recommendations}

Although the positive impact of vitamin D supplementation in improving implantation rates and pregnancy outcomes in ICSI procedures, there is no unequivocal evidence to recommend the routine screening for vitamin $\mathrm{D}$ deficiency 
and its supplementation prior to ICSI and so more randomized controlled trials should be done.

\section{Conflicts of Interest}

The authors declare that they have no conflict of interest.

\section{References}

[1] Thoma, M.E., McLain, A.C., Louis, J.F., King, R.B., Trumble, A.C., Sundaram, R. and Buck Louis, G.M. (2013) Prevalence of Infertility in the United States as Estimated by the Current Duration Approach and a Traditional Constructed Approach. Fertility and Sterilty, 99, 1324-1331.e1. https://doi.org/10.1016/j.fertnstert.2012.11.037

[2] Sunderam, S., Kissin, D.M., Crawford, S.B., Folger, S.G., Jamieson, D.J. and Barfield, W.D. (2014) Assisted Reproductive Technology Surveillance: United States, 2011. MMWR SurveillSumm, 63, 1-28.

[3] Henningsen, A.A., Gissler, M., Skjaerven, R., Bergh, C., Tiitinen, A., Romundstad, L.B., Wennerholm, U.B., Lidegaard, O., Nyboe Andersen, A., Forman, J.L., et al. (2015) Trends in Perinatal Health after Assisted Reproduction: A Nordic Study from the CoNARTaS Group. Human Reproduction, 30, 710-716. https://doi.org/10.1093/humrep/deu345

[4] Rudick, B., Ingles, S., Chung, K., et al. (2012) Characterizing the Influence of Vitamin D Levels on IVF Outcomes. Human Reproduction, 27, 3321-3327. https://doi.org/10.1093/humrep/des280

[5] Dabrowski, F.A., Grzechocinska, B. and Wielgos, M. (2015) The Role of Vitamin D in Reproductive Health: A Trojan Horse or the Golden Fleece? Nutrients, 7, 4139-4153. https://doi.org/10.3390/nu7064139

[6] Anagnostis, P., Karras, S. and Goulis, D.G. (2013) Vitamin D in Human Reproduction: A Narrative Review. International Journal of Clinical Practice, 67, 225-235. https://doi.org/10.1111/ijcp.12031

[7] Aleyasin, A., Hosseini, M.A., Mahdavi, A., et al. (2011) Predictive Value of the Level of Vitamin D in Follicular Fluid on the Outcome of Assisted Reproductive Technology. European Journal of Obstetrics and Gynecology, 159, 132-137. https://doi.org/10.1016/j.ejogrb.2011.07.006

[8] Rainer, D., Davis, E., Peck, J., et al. (2012) Conference Abstracts. Fertility Weekly, 13-17.

[9] Lerchbaum, E and Rabe, T. (2014) Vitamin D and Female Fertility. Current Opinion in Obstetrics and Gynecology, 26, 145-150. https://doi.org/10.1097/GCO.0000000000000065

[10] Aghajafari, F., Nagulesapillai, T., Ronksley, P.E., Tough, S.C., O 'Beirne, M. and Rabi, D.M. (2013) Association between Maternal Serum 25-Hydroxy Vitamin D and Pregnancy and Neonatal Outcomes. BMJ, 346, F1169. https://doi.org/10.1136/bmj.f1169

[11] Miyashita, M., Koga, K., Izumi, G. and Sue, F. (2016) Effect of Vitamin D3 on Endometriosis. The Journal of Clinical Endocrinology \& Metabolism, 101, 2371-2379. https://doi.org/10.1210/jc.2016-1515

[12] Luk, J., Torrealday, S., Neal Perry, G. and Pal, L. (2012) Relevance of Vitamin D in Reproduction. Human Reproduction, 27, 3015-3027. 
https://doi.org/10.1093/humrep/des248

[13] Gittoes, N.J. (2015) Vitamin D: What Is Normal According to Latest Research and How Should We Deal with It? Clinical Medicine, 15, s54-s57.

https://doi.org/10.7861/clinmedicine.15-6-s54

[14] Lerchbaum, E. and Obermayer-Pietsch, B. (2012) Mechanisms in Endocrinology: Vitamin D and Fertility: A Systematic Review. European Journal of Endocrinology, 166, 765-778. https://doi.org/10.1530/EJE-11-0984

[15] Potashnik, G., Lunenfeld, E., Levitas, E., Itskovitz, J., Albutiano, S., et al. (1992) The Relationship between Endogenous Oestradiol and Vitamin D3 Metabolites in Serum and Follicular Fluid during Ovarian Stimulation for in Vitro Fertilization and Embryo Transfer. Human Reproduction, 7, 1357-1360. https://doi.org/10.1093/oxfordjournals.humrep.a137573

[16] Estes, S.J., Ye, B., Qiu, W., Cramer, D., Hornstein, M.D., et al. (2009) A Proteomic Analysis of IVF Follicular Fluid in Women. Fertility and Sterilty, 92, 1569-1578. https://doi.org/10.1016/j.fertnstert.2008.08.120

[17] Anifandis, G.M., Dafopoulos, K., Messini, C.I., et al. (2010) Prognostic Value of Follicular Fluid 25-OH Vitamin D and Glucose Levels in the IVF Outcome. Reproductive Biology and Endocrinology, 8, 91-95.

https://doi.org/10.1186/1477-7827-8-91

[18] Jensen, M.B., Nielsen, J.E., Jørgensen, A., et al. (2010) Vitamin D Receptor and Vitamin D Metabolizing Enzymes Are Expressed in the Human Male Reproductive Tract. Human Reproduction, 25, 1303-1311. https://doi.org/10.1093/humrep/deq024

[19] Li, L., Schriock, E., Dougall, K., et al. (2012) Conference Abstracts. Fertility Weekly, $12,17$.

[20] Gebriel, A.E.M.A., Hassan, F.I., Abdel Latif, E.M., Hassan, E.A. and Aref, M.I. (2017) Effect of Vitamin D Status on Clinical Pregnancy Rate in Case of Intracytoplasmic Sperm Injection (ICSI). Vitamins \& Minerals, 6, 2. https://doi.org/10.4172/2376-1318.1000156

[21] Rainer, D., Davis, E., Peck, J., et al. (2012) Conference Abstracts. Fertility Weekly, 13-17.

[22] Ozkan, S., Jindal, S., Greenseid, K., Shu, J., Zeitlian, G., Hickmon, C. and Pal, L. (2010) Replete Vitamin D Stores Predict Reproductive Success Following in Vitro Fertilization. Fertility and Sterilty, 94, 1314-1319. https://doi.org/10.1016/j.fertnstert.2009.05.019 